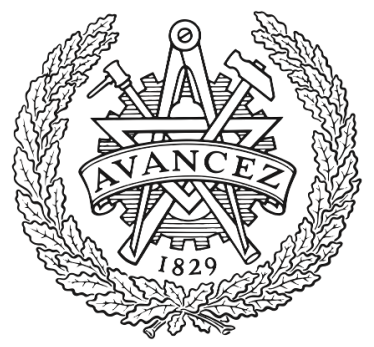

CHALMERS

UNIVERSITY OF TECHNOLOGY

\title{
Efficient spot welding sequence simulation in compliant variation simulation
}

Downloaded from: https://research.chalmers.se, 2023-04-26 12:05 UTC

Citation for the original published paper (version of record):

Sadeghi Tabar, R., Lorin, S., Cromvik, C. et al (2020). Efficient spot welding sequence simulation in compliant variation simulation. ASME International Mechanical Engineering Congress and Exposition, Proceedings (IMECE), 2B-2020. http://dx.doi.org/10.1115/IMECE2020-23798

N.B. When citing this work, cite the original published paper. 


\section{EFFICIENT SPOT WELDING SEQUENCE SIMULATION IN COMPLIANT VARIATION SIMULATION}

\author{
Roham Sadeghi Tabar* \\ Department of Industrial \\ and Materials Science \\ Chalmers University of Technology \\ SE-412 96 Gothenburg \\ Sweden \\ rohams@chalmers.se
}

\author{
Samuel Lorin \\ Christoffer Cromvik \\ Computational Engineering \\ and Design \\ Fraunhofer Chalmers Center \\ SE-412 58 Gothenburg \\ Sweden
}

\author{
Lars Lindkvist \\ Kristina Wärmefjord \\ Rikard Söderberg \\ Department of Industrial \\ and Materials Science \\ Chalmers University of Technology \\ Gothenburg, Sweden
}

\begin{abstract}
Geometric variation is one of the sources of quality issues in a product. Spot welding is an operation that impacts the final geometric variation of a sheet metal assembly considerably. Evaluating the outcome of the assembly, considering the existing geometrical variation between the components can be achieved using the Method of Influence Coefficients (MIC), based on the Finite Element Method (FEM). The sequence, with which the spot welding operation is performed, influences the final geometric deformations of the assembly. Finding the optimal sequence that results in the minimum geometric deformation is a combinatorial problem that is experimentally and computationally expensive. For an assembly with $N$ number of welds, there are $N$ ! possible sequences to perform the spot welding operation. Traditionally, spot welding optimization strategies have been to simulate the geometric variation of the spot-welded assembly after the assembly has been positioned in an inspection fixture, using an appropriate measure of variation. In this approach, the calculation of deformation after springback is one of the most time-consuming steps. In this paper, the cause of variation in the deformations after the springback, between different sequences is identified. The relative displacements of the weld points in the assembly fixture, when welded in a sequence, is the source of such behavior. Capturing these
\end{abstract}

\footnotetext{
*Address all correspondence to this author.
}

displacements leads to large time savings during sequence optimization. Moreover, this approach is independent of the inspection fixture.

The relative weld displacements have been evaluated on two sheet metal assemblies. The sequence optimization problem has been solved for the two assemblies using this approach. The optimal sequence, the corresponding final assembly deformations, and the time-consumption have been compared to the traditional approach. The results show a significant correlation between the weld relative displacements in the assembly fixture, and the assembly deformation in the inspection fixture. Considering the relative weld displacement makes each assembly evaluation less time-consuming, and thereby, sequence optimization time can be reduced up to $30 \%$, compared to the traditional approach.

Keywords: spot welding. sequence. optimization. compliant variation simulation. deformation

\section{INTRODUCTION}

Geometrical variation is the source of the aesthetic and functional problems in the assemblies. The disturbances in the assembly process, and individual components variation, lead to a non-nominal assembly. For compliant assemblies, the joining process is among the critical processes inducing geometrical 
variation in the assemblies. Controlling the joining parameters is a common challenge in the manufacturing industry. The sequence with which the assembly is joined has shown to have a considerable effect on the final geometrical outcome [1-4]. To identify the best joining sequence for the sheet metal assemblies, using variation simulation, is a combinatorial problem. This problem has a costly function evaluation, requiring timeconsuming simulations. The number of possible alternatives to perform welding increases factorially by increasing the number of welds. The need for a more time-efficient and accurate simulation method to determine the optimal joining sequence is preeminent.

\subsection{Compliant Variation Simulation}

Compliant variation simulation is introduced to evaluate the sheet metal assemblies, considering the variation of the component [5]. In this approach, FEM and Monte Carlo (MC) simulations are combined to evaluate the outcome of the assembly considering the geometrical variation in the components, from the corresponding tolerance distributions. To further increase the time efficiency of the method, the Method of Influence Coefficients is introduced [6]. MIC builds linear relationships between the part deviation and acting forces on the assembly. The response of the assembly to these forces is saved in a sensitivity matrix, and associated to the part deviations. The MIC approach is complemented by contact modeling to increase the accuracy of the simulation. The contact modeling avoids the parts to penetrate in the adjacent areas [7,8]. With the small displacements assumption, elastic material, and neglecting the effect of heat, the spot welding process is introduced to the variation simulation $[6,9]$. Based on the approach a multistation assembly perspective has also been developed [10]. To evaluate the effect of joining sequence, the springback of the assembly after each joint is calculated $[11,12]$. The MIC and contact modelling for evaluating the spot welded assemblies has been further improved by removing the intermediate springback calculations [13]. With the state of the art variation simulation, a noticeable fraction of the simulation time is dedicated to springback calculation in the inspection fixture, with respect to different joints. In this paper, a new time-efficient method to simulate the geometrical outcome of the assemblies with respect to the joining sequences is proposed.

\subsection{Spot Welding Sequence Analysis}

Determining the optimal sequence of welds is an NPhard combinatorial problem. The physical experiments for this reason are economically infeasible. Therefore, the optimization algorithms are often combined with a simulation tool for assembly evaluation $[14,15]$. With the state of the art variation simulation, or other FEM based simulations, the most timeconsuming step of the optimization is the assembly evaluation
[2]. To find the optimal sequence with the minimal number of assembly simulations have been addressed in previous studies $[3,16]$. Algorithms based on a random search, such as the genetic algorithm, have been studied extensively [1, 14]. However, these methods are highly dependent on the number of assembly evaluations performed. For Larger population sizes, larger number of assembly simulations are required. More time-efficient rule-based approaches have been introduced using the complaint variation simulation as an evaluator [3]. A surrogate modeling approach has also been introduced, together with an efficient sampling strategy, using MIC and contact modeling. [16]. Deploying compliant variation simulation as an evaluator, a novel stepwise algorithm for joining sequence optimization is also introduced [17]. The previous studies have focused on reducing the number of evaluations by the compliant variation simulation. In this paper, the perspective of reducing each assembly simulation time, from the compliant variation simulation, is taken into consideration.

\subsection{Scope of the Paper}

Spot welding sequence optimization is a time-consuming task. Evaluating the assembly deformation with respect to the part deviations is performed with compliant variation simulation. In this simulation, springback calculation, while the part is not over-constrained in the inspection fixture, is one of the time-consuming steps. To optimize the sequence of welds, a large number of sequences need to be evaluated. Therefore, a more efficient approach for variation simulation with respect to welding sequences are looked after. In this paper, an efficient variation simulation approach for welding sequence optimization is proposed. Section 1 provided an introduction to the problem. Section 2 presents the proposed approach followed by the presentation of the reference assemblies in Section 3 . Section 4 presents the evaluation of the approach on the reference assemblies. Finally, in Section 5, the conclusion are drawn based on the results achieved, and the future research scope is presented.

\section{PROPOSED APPROACH}

In this section, the proposed variation simulation approach for the spot welded assemblies, considering the sequence of welding, is introduced. The standard, state of the art, variation simulation is introduced in Section 2.1. Sections 2.2 to 2.4 present the proposed approach.

\subsection{Assembly Simulation Steps}

There are two aspects constructing the final assembly deviation. These are, part deviations from previous manufacturing steps, $\boldsymbol{u}^{d e v}$, and assembly deformation $\boldsymbol{u}$. The steps below describe how to calculate the assembly deformation 
while the part is held in the assembly fixture. The general steps of the assembly modeling in variation simulation, following the formulation in [18], include:

1. Positioning parts and clamping in the fixture.

2. Derive the clamping, contact, and joining forces, $f_{c l}^{0}, f_{c}^{0}$, and $f_{w}^{0}$ respectively. Here, contact modeling is used. It has to be noted that, $\boldsymbol{f}_{w}^{0}$, and $\boldsymbol{f}_{c}^{0}$ can be calculated directly using the contact modeling, but $\boldsymbol{f}_{c l}^{0}$ is calculated successively, see [18]. Expressing the initial stiffness matrix ${ }^{1}$ with $\boldsymbol{K}^{\mathbf{0}}$, and the assembly deformation as $\boldsymbol{u}^{0}$, then the following holds:

$$
\boldsymbol{K}^{\mathbf{0}} \boldsymbol{u}^{\mathbf{0}}=\boldsymbol{f}_{c l}^{0}+\boldsymbol{f}_{c}^{\mathbf{0}}+\boldsymbol{f}_{w}^{0}
$$

Since the number of non-zero elements in $f_{c l}^{0}, f_{c}^{0}$, and $f_{w}^{0}$ are typically relatively small, the corresponding sensitivity matrix, which consists of the corresponding column in the inverse matrix, is pre-calculated. The relation $\boldsymbol{S}^{\mathbf{0}}=\left[\boldsymbol{K}^{\mathbf{0}}\right]^{-1}$ holds only with respect to the relevant rows [6]. Hence, the following can be written:

$$
\boldsymbol{u}^{\mathbf{0}}=\boldsymbol{S}^{\mathbf{0}}\left(\boldsymbol{f}_{c l}^{\mathbf{0}}+\boldsymbol{f}_{c}^{\mathbf{0}}+\boldsymbol{f}_{w}^{\mathbf{0}}\right)
$$

To achieve the contact equilibrium, the contact forces are calculated using quadratic programming [7]. Finding the contact forces is a non-linear problem imposing non-linear behavior to the equation above.

3. Joining parts through adding stiff beams, locking all the degrees of freedom between the weld pairs ${ }^{2}$. Expressing the updated stiffness modifier as $\Delta$, that is the stiffness of the beam, then:

$$
\left(\boldsymbol{K}^{\mathbf{0}}+\Delta \boldsymbol{K}^{\mathbf{0}}\right) \boldsymbol{u}^{\mathbf{0}} \equiv \boldsymbol{K}^{\mathbf{1}} \boldsymbol{u}^{\mathbf{0}}=\boldsymbol{f}_{c l}^{\mathbf{0}}+\boldsymbol{f}_{c}^{0}
$$

or

$$
u^{0}=S^{1}\left(f_{c l}^{0}+f_{c}^{0}\right)
$$

where the relation

$$
\Delta \boldsymbol{K}^{0} \boldsymbol{u}^{\mathbf{0}}=-\boldsymbol{f}_{w}^{0}
$$

is used.

\footnotetext{
${ }^{1}$ modified to account for boundary conditions

${ }^{2}$ weld pairs is one node on each part that define where the spot weld is added
}

4. Next, the contact forces are removed, and the springback is calculated

$$
\boldsymbol{u}_{s b}^{1}=-\boldsymbol{S}^{1} \boldsymbol{f}_{c} .
$$

The new penetrated state after one weld is,

$$
u^{1}=u^{0}+u_{s b}^{1}
$$

5. From this step, new clamping, contact, and weld forces are calculated, as in step 1 , using the sensitivity matrix $S^{1}$.

6. Iterate among the welds in a sequence. Using the sensitivity matrix $\boldsymbol{S}^{i}$ at each welding step $i$, for an assembly with $n$ spot welds, the aggregated deformation after welding in a sequence is calculated as:

$$
\boldsymbol{u}^{n}=\boldsymbol{u}^{\mathbf{0}}+\sum_{i=1}^{n} \boldsymbol{S}^{i} \boldsymbol{f}_{c}^{i}
$$

After these steps, the assembly is typically placed in an inspection fixture, where the it is not over-constrained. To calculate the final deformation in this fixture, new sensitivity matrices, a new penetration state, and new penetration forces need to be calculated.

However, assuming elasticity, which steps 1-5 above are based on, given the relative displacement in every weld pair, that is locked after welding, the final displacement can be calculated. This is achieved by positioning every part in the inspection fixture, constraining each weld pair to be in the relative displacement found during spot welding in the assembly fixture, adding corresponding beams, and calculating the final shape, including the resolved penetration by contact modeling.

Since calculating the springback in the inspection fixture is time-consuming, considering that the relative displacements are captured in the assembly fixture, this information can be used to construct the deviations in the inspection points. Finding the optimal sequence of welding with respect to these relative displacements in the assembly fixture can reveal the optimal sequence in the inspection fixture, without having to calculate the last springback step.

\subsection{Relative Displacements}

For weld sequence optimization with respect to the assembly deformation, after applying $n$ weld points, Equation 8, reduced assembly simulation time is searched after. Since the assembly deformation, in the inspection fixture, depends on the assembly fixture, the relative displacements in the weld points, and the 


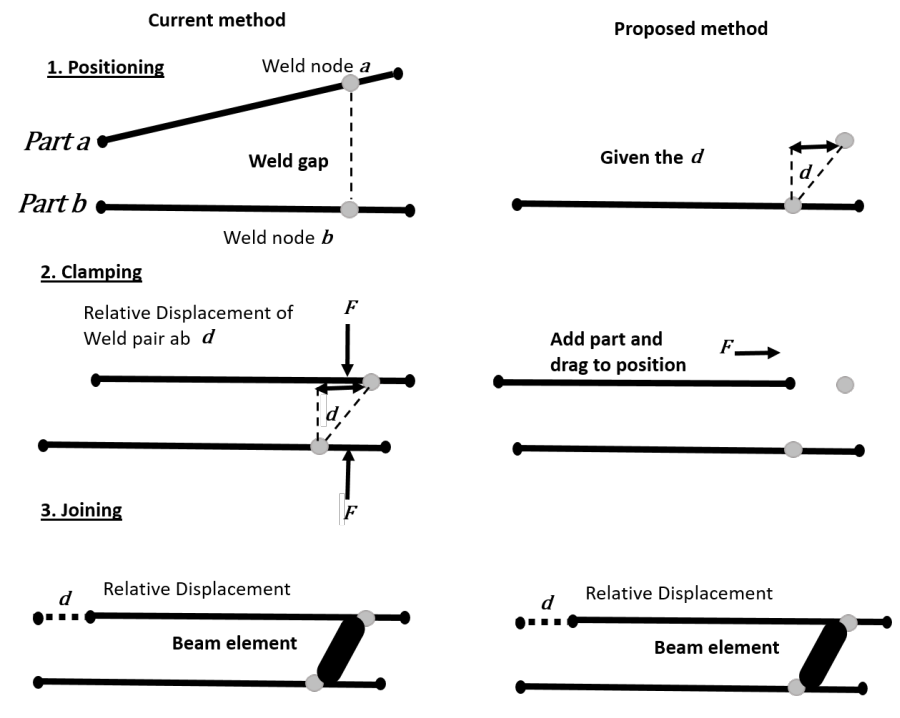

FIGURE 1. Relative displacement between the weld pairs

contact forces, it is expected that the sequence of welds in the assembly fixture with the smallest relative displacements in the weld points, represent the assembly with the lowest total deformation.

Fig. 1 is the schematic view of the calculation procedure of weld relative displacements, $d$. On the left side, the current procedure is visualized. For non-nominal parts, the parts are positioned in the assembly fixture; forces are applied to mate the parts in the welding nodes. These forces cause the parts to move relative to each other. Finally, to join the parts, a stiff beam element is added, locking all the degrees of freedom on the weld pairs together. From another perspective, if the relative displacements in the clamped position is known, it is expected that these relative displacements be directly translated to the inspection fixture. On the right side of Fig 1, the proposed modifications are visualized. Given the relative displacements in the clamping position, the deformation after spring back can be calculated by adding a part and dragging the weld nodes to the calculated relative displacements. The joint can be defined in the calculated position. Therefore, if the relative displacements in all the welds are captured, while each weld is set in a sequence, these displacements should describe the behavior between the sequences.

\subsection{Correlation Analysis}

To show the dependency of the weld relative displacements in the assembly fixture, $d$, and the total assembly deformation in the inspection fixture, the correlation of the two variables are analyzed. Let us define the total assembly deformation, $\boldsymbol{u}+\boldsymbol{u}^{\text {dev }}$ in the inspection fixture, as the Root Mean Square (RMS) of the magnitude of displacements in each mesh node for a specific sequence $i$, as $u_{i}$. Considering that there are $r$ nodes in the assembly, then

$$
A_{m}^{R M S}=\sqrt{\frac{1}{r} \sum_{r}\left\|u_{i}^{r}\right\|^{2}}
$$

$A_{m}^{R M S}$ depends on the contact forces in the inspection fixture, and hence is a function of the initial deviation (incoming part deviation). It also depends on the assembly fixture, and relative displacements in the weld points. Now the Root Sum Square (RSS) of the relative displacements $d$ for that specific sequence, $i$ with $n$ weld points is:

$$
A_{d}^{R S S}=\sqrt{\sum_{n} d_{i}^{n 2}}
$$

The correlation of the two is calculated as:

$$
\rho_{A_{m}^{R M S}}, A_{d}^{R S S}=\frac{E\left[\left(A_{m}^{R M S}-\mu_{A_{m}^{R M S}}\right)\left(A_{d}^{R S S}-\mu_{A_{d}^{R S S}}\right)\right]}{\sigma_{A_{m}^{R M S}} \sigma_{A_{d}^{R S S}}} .
$$

Given the exact $A_{d}^{R S S}$ values, the assembly can be put in any inspection fixture without recalculating all the steps mentioned in Section 2.1. However, to calculate $A_{m}^{R M S}$ all the steps must be followed. Showing that the relative displacements in the welds and the assembly deformation measure have a significant positive correlation determines that the two measures can be used as an objective for sequence optimization interchangeably.

\subsection{Sequence Optimization}

Showing that the relative displacements of the assembly weld points, set in a sequence in the assembly fixture, and the total assembly deformation in the inspection fixture, are highly correlated, this measure can be used as the objective of the sequence optimization. To capture the relative displacements $d$, the springback calculation in the inspection fixture, Fig. 1, do not need to be performed. These displacements can be captured after clamping and setting each weld in a sequence. This is a prominent advantage for sequencing, where a large fraction of the simulation time can be bypassed. The minimization of the relative displacements $A_{d}^{R S S}$ among the sequences, for an assembly with $n$ welds with the sequence $W_{i}=\left[x_{1}, \ldots, x_{n}\right]$, can 
be formulated as:

$$
\begin{array}{cl}
\underset{W_{i}}{\operatorname{minimize}} & A_{d}^{R S S}\left(W_{i}\right) \\
\text { subject to } & \boldsymbol{W}:\{1, \ldots, n\} \rightarrow\{n, \ldots, 1\}, n \in \mathbb{N} \\
& W_{i} \subseteq \boldsymbol{W}, i \in \mathbb{N}: 1 \leq i \leq|\boldsymbol{W}| \\
& W_{i}=\left\{x_{i 1}, \ldots, x_{i j}\right\}, x_{i j} \in \mathbb{N}: 1 \leq j \leq n \\
& \left|W_{i}\right|=n .
\end{array}
$$

Only complete permutations of 1 to $n$, are to be considered in the solution space. To solve the optimization problem the stepwise algorithm proposed in [17], is used. The state-space search approach in this algorithm is based on evaluation of all the possible alternatives for each sequence element, while the rest of the weld points are set. With this algorithm, the optimized sequence with respect to the assembly deformation, and weld relative displacements are derived and compared. The total optimization time using each method is also evaluated and compared.

\section{REFERENCE ASSEMBLIES}

Two sheet metal assemblies are evaluated with the proposed approach, the correlation between the relative displacement of welds set in a sequence, and the assembly deformation measure is analyzed. The details of each assembly are provided in this section.

\subsection{Assembly A}

Assembly A is composed of two parts with seven weld points. The CAT model is prepared in the CAT-tool RD\&T [19]. The positioning system, the weld points, and their corresponding numbering are shown in Fig. 2. To evaluate the geometrical deformation of the assembly after springback in the inspection fixture, with respect to a specific sequence, 7.28 seconds is required. Evaluating the relative displacements of the welds for each sequence in the assembly fixture requires 5.65 seconds. The evaluation times are calculated using a workstation with 2.7 GHz CPU, and $32 \mathrm{~GB}$ RAM. The correlation analysis and optimization results are presented in the Section 4.1.

\subsection{Assembly B}

This assembly is composed of three parts with five weld points. The CAT model is prepared, and the contact modeling and the part deviation input are applied to the model, same as Assembly A. The CAT model of the assembly is presented in Fig. 3. To evaluate the assembly geometrical deviation in the inspection fixture, welded in a sequence, 7.63 seconds is required. Evaluating the relative displacements of the welds

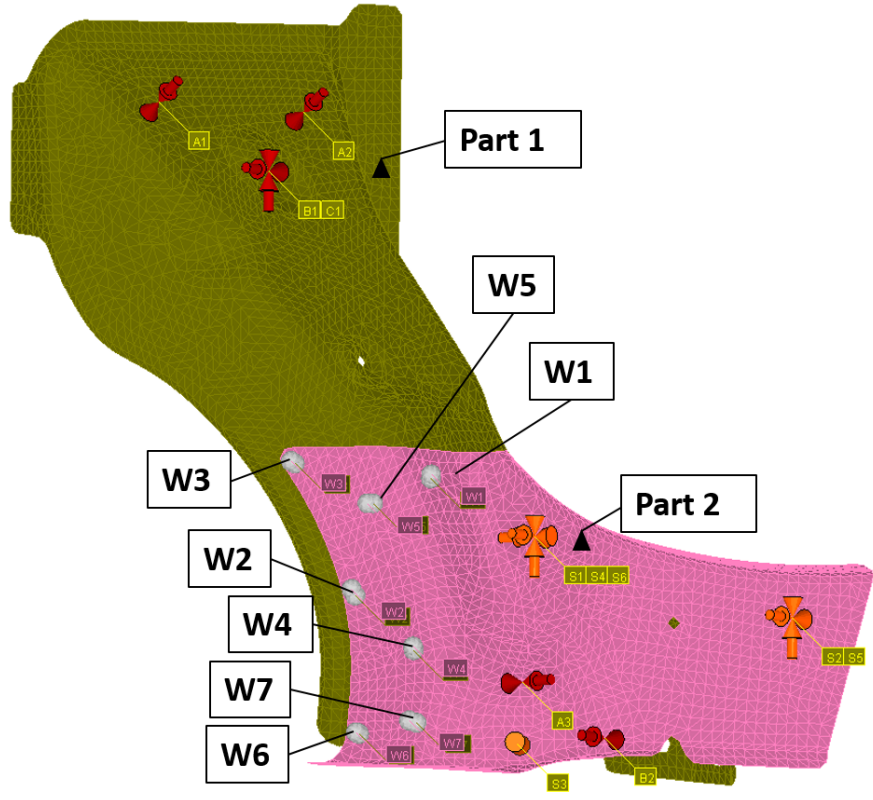

FIGURE 2. Assembly A

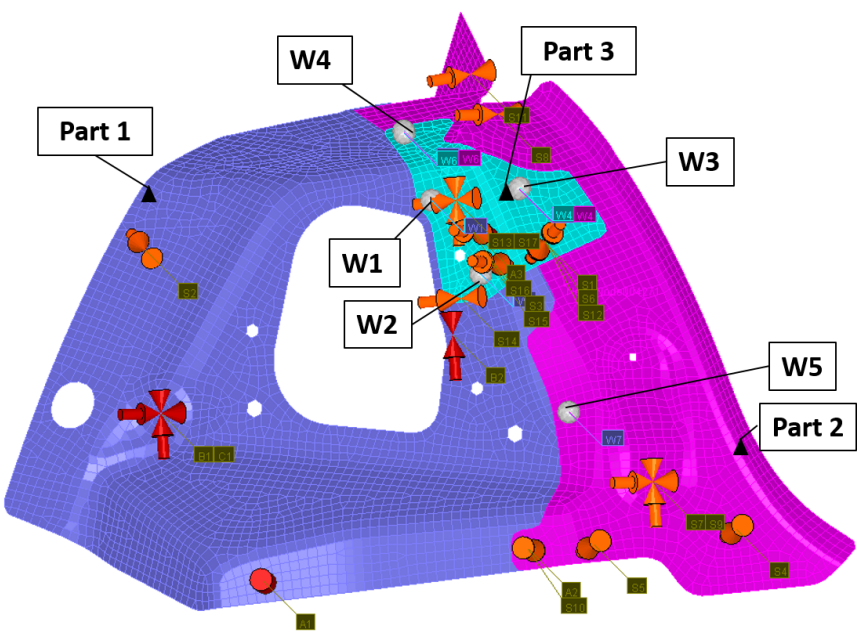

FIGURE 3. Assembly B

in the assembly fixture, requires 5.27 seconds, using the same workstation as Assembly A.

\section{ASSEMBLY EVALUATION}

The sequence optimization with respect to the relative displacements of the welds, $A_{d}^{R S S}$, and RMS of the deformations in all the assembly nodes, $A_{m}^{R M S}$ are performed on both the reference assemblies and the retrieved sequence, and the 


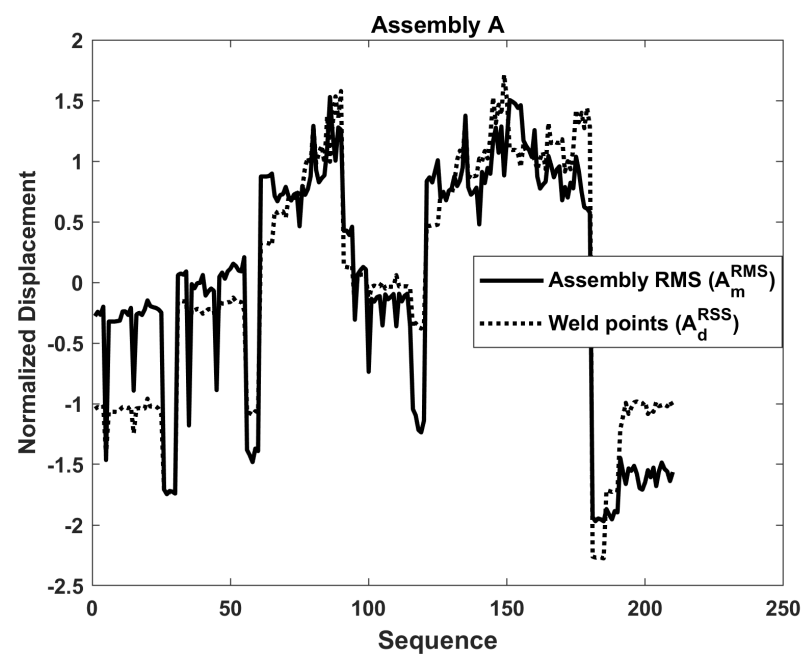

FIGURE 4. Assembly A: Comparison between $A_{d}^{R S S}$ and, $A_{m}^{R M S}$

optimization time are compared. The detailed results for each assembly are presented in this section.

\subsection{Assembly A}

To compare the optimization objectives, the two measures $A_{d}^{R S S}$, and $A_{m}^{R M S}$ are normalized, for all the combinations of the first three sequence elements. Fig. 4 shows the behavior of the two measures among the sequences. The two measures follow the same trend. The proposed method and the corresponding measure, $A_{d}^{R S S}$ is capturing the sequence with the minimum interval. To get an understanding of the correlation of the two measures, a correlation analysis is performed. Fig 5, shows the one by one linear correlation of the two measures. As can be seen, the $\rho$ value of 0.91 specifies a significant positive correlation between the two.

Optimization of the sequences with respect to the measures are conducted using the stepwise algorithm, Section 2.4. The optimization results are reported in Table 1. Optimization with respect to the two objectives, results in the identical sequences, with the assembly deformation of $0.4289 \mathrm{~mm}$. Considering the optimization time required in each method, the relative displacement of the welds as the objective, helps to save $22.39 \%$ of the optimization time, compared to the total assembly deformation RMS optimization.

\subsection{Assembly B}

The same analysis has been performed for this assembly. Since the assembly has five weld points, all the possible 120 sequences are evaluated, and the sequence that corresponds to the minimum assembly deformation is identified. Fig. 6 shows the normalized RMS of the magnitude of displacements for

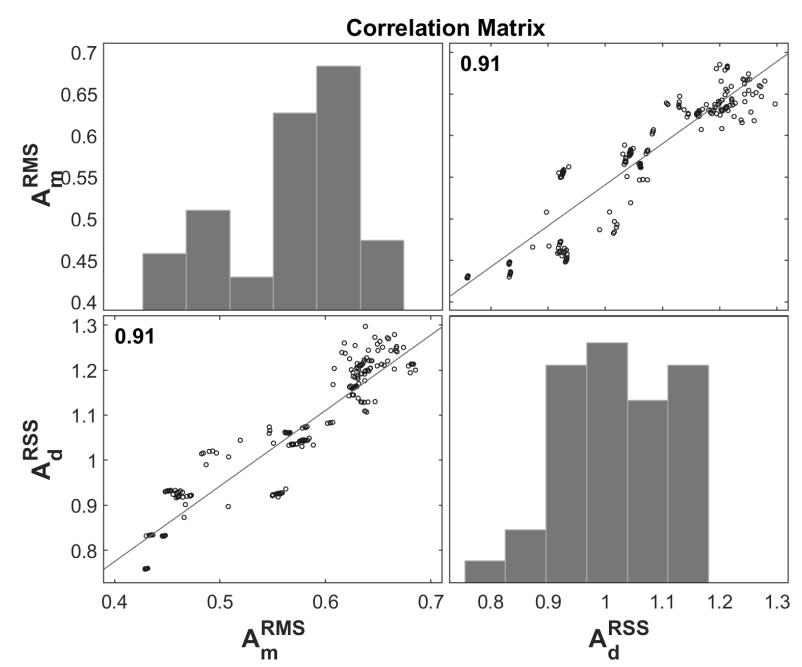

FIGURE 5. Assembly A: Correlation between $A_{d}^{R S S}$ and, $A_{m}^{R M S}$

TABLE 1. SEQUENCE OPTIMIZATION RESULTS

\begin{tabular}{ccccc}
\hline Assembly & Method & $\begin{array}{c}\text { Optimal } \\
\text { Sequence }\end{array}$ & $\begin{array}{c}\text { Assembly } \\
\text { Deformation }\end{array}$ & $\begin{array}{c}\text { Optimization } \\
\text { time }\end{array}$ \\
\hline A & $A_{m}^{R M S}$ & {$[1,7,2,6,5,4,3]$} & 0.4289 & 1594.3 \\
A & $A_{d}^{R S S}$ & {$[1,7,2,6,5,4,3]$} & 0.4289 & 1237.3 \\
B & $A_{m}^{R M S}$ & {$[2,1,4,5,3]$} & 1.0689 & 915.6 \\
B & $A_{d}^{R S S}$ & {$[2,1,4,5,3]$} & 1.0689 & 632.4 \\
\hline
\end{tabular}

all the nodes, in the assembly against, the RSS of the relative weld displacements, for all the sequences. Both measures follow the same trend in this assembly as well. The correlation value $\rho$ of 0.95 also indicates a significant positive correlation between the two, Fig. 7. The sequence corresponding to the minimum assembly deformation is $[2,1,4,5,3]$. Considering the minimization of the proposed approach measure $A_{d}^{R S S}$, results in an identical sequence as total assembly deviation minimization. The optimization time required to achieve the optimum sequence is $30.93 \%$ lower than optimization considering the RMS of the magnitude of the displacements for all the nodes. The summary of the results achieved for this assembly is presented in Table 1 .

\section{CONCLUSION}

Spot welding sequence optimization is a combinatorial problem with an expensive to evaluate objective function. 


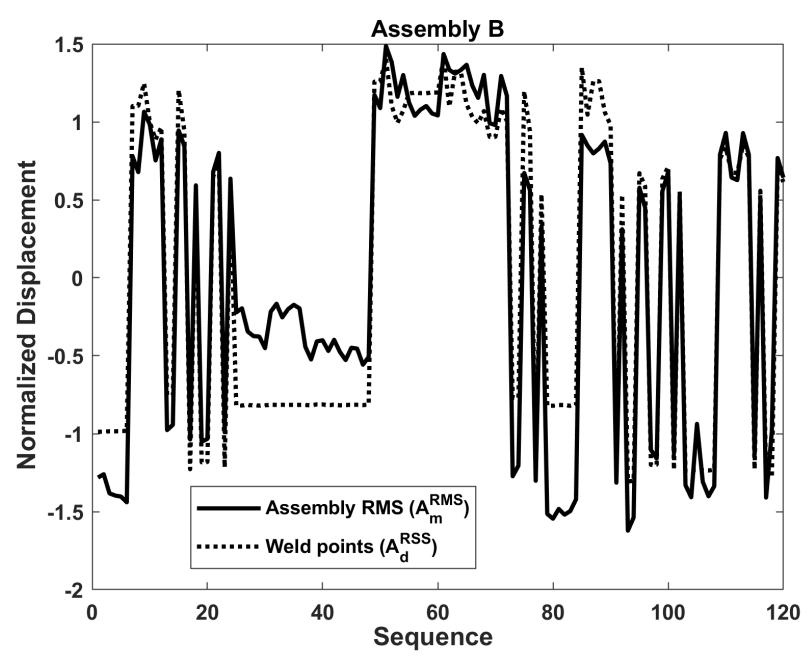

FIGURE 6. Assembly B: Comparison between $A_{d}^{R S S}$ and, $A_{m}^{R M S}$

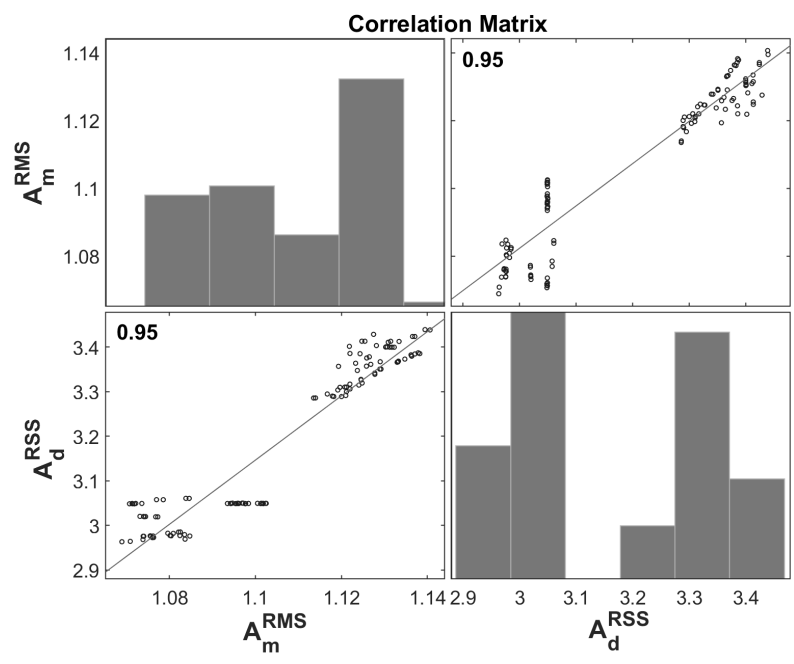

FIGURE 7. Assembly B: Correlation between $A_{d}^{R S S}$ and, $A_{m}^{R M S}$

Considering the part deviation, compliant variation simulation is performed to determine the geometrical outcome of the assembly with a specific welding sequence. This simulation method is based on deformation calculation in different steps. Springback calculation in the inspection fixture is one of the time-consuming steps of this simulation method. In this paper, an efficient spot welding sequence simulation in compliant variation simulation is introduced. The method is based on the calculation of relative displacements of the weld points in the assembly fixture. The method helps to bypass the springback calculation in the inspection fixtures for sequence optimization. The proposed approach has been applied to two sheet metal assemblies, and the sequence optimization is performed. Two approaches have been considered in the comparison of the retrieved results.

1. Sequence optimization with respect to the RMS of the magnitude of the displacements of all the nodes in the assembly in the inspection fixture, $A_{m}^{R M S}$.

2. Sequence optimization with respect to the RSS of the relative displacements of the welds in the assembly fixture, $A_{d}^{R S S}$.

The results show that the proposed method, with the measure of relative displacements in the welds, have a significant positive correlation to the magnitude of the displacements in the assembly. The optimization results indicate that optimizing the welding sequence with respect to the relative displacements of the welds, converges to the identical sequence to the magnitude of displacements in the inspection fixture. Furthermore, it has been shown that the total optimization time with the proposed method is improved up to $30 \%$.

Future research includes expanding the proposed approach with a multivariate analysis approach, where the displacement direction is tracked by the relative displacement of the welds, and thereby, deformation direction in the assembly is correlated to relative displacements in the welds.

\section{ACKNOWLEDGMENT}

The work was carried out in collaboration with Wingquist Laboratory and the Area of Advance Production at Chalmers within the project Smart Assembly 4.0, financed by The Swedish Foundation for Strategic Research. The support is gratefully acknowledged.

\section{REFERENCES}

[1] Tabar, R. S., Wärmefjord, K., and Söderberg, R., 2018. "Evaluating evolutionary algorithms on spot welding sequence optimization with respect to geometrical variation". Procedia CIRP, 75, pp. 421 - 426. The 15th CIRP Conference on Computer Aided Tolerancing, CIRP CAT 2018, 11-13 June 2018, Milan, Italy.

[2] Tabar, R. S., Wärmefjord, K., and Söderberg, R., 2019. "A method for identification and sequence optimisation of geometry spot welds in a digital twin context". Proceedings of the Institution of Mechanical Engineers, Part C: Journal of Mechanical Engineering Science, 233(16), pp. 56105621.

[3] Tabar, R. S., Wärmefjord, K., Söderberg, R., and Lindkvist, L., 2019. "A Novel Rule-Based Method for Individualized Spot Welding Sequence Optimization With Respect to Geometrical Quality". Journal of Manufacturing Science and Engineering, 141(11), 10. 111013. 
[4] Wärmefjord, K., Söderberg, R., and Lindkvist, L., 2010. "Strategies for Optimization of Spot Welding Sequence With Respect to Geometrical Variation in Sheet Metal Assemblies". Vol. Volume 3: Design and Manufacturing, Parts $\mathrm{A}$ and $\mathrm{B}$ of ASME International Mechanical Engineering Congress and Exposition, pp. 569-577. Vancouver, British Columbia, Canada. November 12-18.

[5] Söderberg, R., Lindkvist, L., Wärmefjord, K., and Carlson, J. S., 2016. "Virtual geometry assurance process and toolbox". Procedia CIRP, 43, pp. 3 - 12. 14th CIRP CAT 2016 - CIRP Conference on Computer Aided Tolerancing, 18-20 May 2016, Gothenburg, Sweden.

[6] Liu, S. C., and Hu, S. J., 1997. "Variation simulation for deformable sheet metal assemblies using finite element methods". Journal of Manufacturing Science and Engineering, 119, pp. 368-374.

[7] Lindau, B., Lorin, S., Lindkvist, L., and Söderberg, R., 2016. "Efficient Contact Modeling in Nonrigid Variation Simulation". Journal of Computing and Information Science in Engineering, 16(1), pp. 11002-11007.

[8] Lupuleac, S., Zaitseva, N., Stefanova, M., Berezin, S., Shinder, J., Petukhova, M., and Bonhomme, E., 2019. "Simulation of the Wing-to-Fuselage Assembly Process". Journal of Manufacturing Science and Engineering, 141(6), 04.

[9] Stefanova, M., Yakunin, S., Petukhova, M., Lupuleac, S., and Kokkolaras, M., 2018. "An interior-point methodbased solver for simulation of aircraft parts riveting". Engineering Optimization, 50(5), pp. 781-796.

[10] Camelio, J., Hu, S. J., and Ceglarek, D., 2004. "Modeling Variation Propagation of Multi-Station Assembly Systems With Compliant Parts ". Journal of Mechanical Design, 125(4), 01, pp. 673-681.

[11] Wärmefjord, K., Söderberg, R., and Lindkvist, L., 2010. "Variation Simulation of Spot Welding Sequence for Sheet Metal Assemblies". In Proceedings of NordDesign2010 International Conference on Methods and Tools for Product and Production Development, Vol. 2, pp. 519-528. August 25-27, Gothenburg, Sweden.

[12] Lupuleac, S., Petukhova, M., Shinder, Y., and Bretagnol, B., 2011. Methodology for solving contact problem during riveting process, oct.

[13] Lorin, S., Lindau, B., Lindkvist, L., and Söderberg, R., 2018. "Efficient Compliant Variation Simulation of SpotWelded Assemblies". ASME. J. Comput. Inf. Sci. Eng., 19(1), pp. 011007-011007-7.

[14] Liao, Y. G., 2005. "Optimal design of weld pattern in sheet metal assembly based on a genetic algorithm". The International Journal of Advanced Manufacturing Technology, 26(5), Sep, pp. 512-516.

[15] Xie, L. S., and Hsieh, C., 2002. "Clamping and welding sequence optimisation for minimising cycle time and assembly deformation". International Journal of Materials and Product Technology, 17(5-6), 10, pp. 389-399.

[16] Tabar, R. S., Wärmefjord, K., and Söderberg, R., 2020. "A new surrogate model-based method for individualized spot welding sequence optimization with respect to geometrical quality". The International Journal of Advanced Manufacturing Technology, 106(5), pp. 23332346.

[17] Tabar, R. S., Wärmefjord, K., Söderberg, and Rikard, 2020. "Rapid Sequence Optimization of Spot Welds for Improved Geometrical Quality Using a Novel Stepwise Algorithm”. Engineering Optimization, 0(0), pp. 1-18.

[18] Lorin, S., Lindau, B., Sadeghi Tabar, R., Lindkvist, L., Wärmefjord, K., and Söderberg, R., 2018. "Efficient Variation Simulation of Spot-Welded Assemblies". Vol. Volume 2: Advanced Manufacturing of ASME International Mechanical Engineering Congress and Exposition, p. V002T02A110. Pittsburgh, Pennsylvania, USA.

[19] RD\&T Technology AB, 2017. RD\&T Software Manual. 